\title{
Cauchy Problem of the Singularly Perturbed Sixth Order Boussinesq Type Equation
}

\author{
Hong Li, Changming Song, Li Chen \\ College of Science, Zhongyuan University of Technology, Zhengzhou, China \\ Email: cmsongh@163.com
}

How to cite this paper: Li, H., Song, C.M. and Chen, L. (2017) Cauchy Problem of the Singularly Perturbed Sixth Order Boussinesq Type Equation. Journal of Applied Mathematics and Physics, 5, 1648-1657. https://doi.org/10.4236/jamp.2017.59137

Received: July 20, 2017

Accepted: September 12, 2017

Published: September 15, 2017

\begin{abstract}
In this paper, the existence and uniqueness of the global generalized solution and the global classical solution for the Cauchy problem of the singularly perturbed sixth order Boussinesq type equation are proved.
\end{abstract}

\section{Keywords}

Boussinesq Equation, Cauchy Problem, Global Generalized Solution, Global Classical Solution

\section{Introduction}

In this paper, we consider the following Cauchy problem

$$
\begin{gathered}
u_{t t}=u_{x x}+\sigma(u)_{x x}+\alpha u_{x^{4}}+\beta u_{x^{6}}, x \in R, t>0, \\
u(x, 0)=u_{0}(x), u_{t}(x, 0)=u_{1}(x), x \in R,
\end{gathered}
$$

where $u(x, t)$ is the unknown function, subscript $x$ and $t$ indicate partial derivatives, $\sigma(s)$ is the given function, $\alpha>0$ and $\beta>0$ are real numbers, $u_{0}(x)$ and $u_{1}(x)$ are given functions defined on $R$.

There are also several equations which are closely related to Equation (1.1). In the numerical study of the ill-posed Boussinesq equation

$$
u_{t t}=u_{x x}+\left(u^{2}\right)_{x x}+u_{x x x x}
$$

In [1], Darapi and Hua proposed the singularly perturbed Boussinesq equation

$$
u_{t t}=u_{x x}+\left(u^{2}\right)_{x x}+u_{x x x x}+\delta u_{x x x x x x}
$$

as a dispersive regularization of the ill-posed classical Boussinesq Equation (1.3), where $\delta>0$ is small parameter. The authors use both filtering and regularization techniques to control growth of errors and to provide better approximate 
solutions of this equation.

Dash and Daripi presented a formal derivation of (1.4) from two-dimensional potential flow equations for water waves through an asymptotic series expansion for small amplitude and long wave length in [2] [3]. The physical relevance of equation (1.4) describes the bi-directional propagation of small amplitude and long capillary-gravity waves on the surface of shallow water for bond number (surface tension parameter) less than but very close to $1 / 3$.

In [4], Feng investigated the generalized Boussinesq equation including the singularly perturbed Boussinesq equation

$$
u_{t t}=[Q(u)]_{x x}+\sum_{i=1}^{n} b_{i} u_{(2 i+2) x},
$$

where $Q(u)=u+b_{0} u^{r}, r$ and $b_{i}(i=1, \cdots, n)$ are all real constants. By the means of two proper ansatzs, the author obtained explicit traveling solitary wave solutions.

In [5], Song et al. studied the existence and uniqueness of the global generalized solution and the global classical for the initial boundary value problem of Equation (1.1). In [6], Song et al. also studied the nonexistence of the global solutions for the initial boundary value problem of Equation (1.1).

The aim of the present article is to prove that, by virtue of the Galerkin method and prior estimates, the problem (1.1), (1.2) has a unique global generalized solution and a unique global classical solution.

In order to prove that the Cauchy problem (1.1), (1.2) has a unique global solution, we shall consider the following auxiliary problem

$$
\begin{gathered}
v_{t t}=v_{x x}+\sigma\left(v_{x}\right)_{x}+\alpha v_{x^{4}}+\beta v_{x^{6}}, x \in R, t>0, \\
v(x, 0)=v_{0}(x), v_{t}(x, 0)=v_{1}(x), x \in R .
\end{gathered}
$$

First of all, we shall prove that the periodic boundary value problem of Equation (1.6) has a unique global solution by the Galerkin method. Next, we prove that the Cauchy problem (1.6), (1.7) has a unique global solution by constructing a sequence of periodic boundary value problem of Equation (1.6). Then, we can obtain a unique global solution of the Cauchy problem (1.1), (1.2) from (1.6), (1.7) by setting $v_{x}(x, t)=u(x, t), v_{0 x}(x)=u_{0}(x)$ and $v_{1 x}(x)=u_{1}(x)$.

\section{Periodic Boundary Value Problem of (1.6), (1.7)}

To obtain the global solution for the Cauchy problem (1.6), (1.7), we first discuss the following periodic boundary value problem on $\bar{Q}_{T}$

$$
\begin{gathered}
v_{t t}=v_{x x}+\sigma\left(v_{x}\right)_{x}+\alpha v_{x^{4}}+\beta v_{x^{6}}, \\
v_{x}(x, t)=v_{x}(x+2 D, t), \\
v(x, 0)=v_{0}(x), v_{t}(x, 0)=v_{1}(x),
\end{gathered}
$$

where $\bar{Q}_{T}=\bar{\Omega} \times[0, T], \bar{\Omega}=[-D, D], D>0, v_{0}(x)$ and $v_{1}(x)$ are given functions defined on $\bar{\Omega}$ and satisfy (2.2). 
Let $\left\{y_{n}(x)\right\}=\left\{\frac{1}{\sqrt{2 D}}, \frac{1}{\sqrt{D}} \cos \delta_{n} x, \frac{1}{\sqrt{D}} \sin \delta_{n} x, n=1,2, \cdots\right\}$ be the orthogonal base in $L^{2}(\Omega)$ composed of the eigenfunctions of the eigenvalue problem

$$
\begin{aligned}
& y^{\prime \prime}+\lambda y=0, x \in \Omega, \\
& y_{x}(x)=y_{x}(x+2 D)
\end{aligned}
$$

corresponding to eigenvalue $\lambda_{i}=\delta_{i}^{2}=\left(\frac{i \pi}{D}\right)^{2}(i=1,2, \cdots)$. Let

$$
v_{N}(x, t)=\sum_{i=1}^{N} \alpha_{N i}(t) y_{i}(x)
$$

be the Galerkin approximate solution of the problem (2.1)-(2.3), where $\alpha_{N i}(t)$ are the undermined functions, $N$ is a natural number.

Substituting $v_{N}(x, t)$ and the approximations $v_{0}(x), v_{1}(x)$ into the problem (2.1)-(2.3), we get

$$
\begin{gathered}
v_{N t t}=v_{N x x}+\sigma\left(v_{N x}\right)_{x}+\alpha v_{N x^{4}}+\beta v_{N x^{6}}, \\
v_{N x}(x, t)=v_{N x}(x+2 D, t), \\
v_{N}(x, 0)=v_{0 N}(x), v_{N t}(x, 0)=v_{1 N}(x) .
\end{gathered}
$$

Multiplying both sides of (2.4) and (2.6) by $y_{s}(x)$, summing up for $s=1,2, \cdots, N$ and integrating on $\Omega$, we have

$$
\begin{gathered}
\left(v_{N t t}-v_{N x x}-\sigma\left(v_{N x}\right)_{x}-\alpha v_{N x^{4}}-\beta v_{N x^{6}}, y_{s}\right)=0, \\
v_{N s}(0)=\beta_{s}, v_{N s t}(0)=\gamma_{s}, s=1,2, \cdots, N .
\end{gathered}
$$

Lemma 2.1. (Adams [7]) There exist constants $\varepsilon>0$ and $C(\varepsilon)>0$ such that for any integers $j$ and $m, 0 \leq j \leq m$, the following inequality holds

$$
\left\|D_{x}^{j} u\right\|^{2} \leq C(\varepsilon)\|u\|^{2}+\varepsilon\left\|D_{x}^{m} u\right\|^{2} .
$$

Lemma 2.2. Assume that $\sigma \in C^{1}(R), \sigma^{\prime}(s)$ is bounded blow, i.e., there is a constant $C_{0}$ such that $\sigma^{\prime}(s) \geq C_{0}$ for any $s \in R, v_{0} \in H^{3}(\Omega), v_{1} \in L^{2}(\Omega)$. Then for any $N$, the problem (2.7), (2.8) has a global classical solution $\alpha_{N s}(t) \in C^{2}[0, T] \quad(s=1,2, \cdots, N)$. Moreover, we have the following estimate

$$
\left\|v_{N}(\cdot, t)\right\|_{H^{3}(\Omega)}^{2}+\left\|v_{N t}(\cdot, t)\right\|^{2} \leq C_{1}(T), t \in[0, T],
$$

where and in the sequel $C_{i}(T)(i=1,2, \cdots)$ are constants which depend on $T$, but not on $N$ and $\Omega$.

Proof: Let $\sigma_{1}(s)=\sigma(s)-k_{0} s-\sigma(0), k_{0}=\min \left\{C_{0}, 0\right\} \leq 0$, then $\sigma_{1}(0)=0$, $\sigma_{1}^{\prime}(s)=\sigma^{\prime}(s)-k_{0} \geq 0$ and $\sigma_{1}(s)$ is a monotonically increasing function, and thus

$$
\int_{0}^{s} \sigma_{1}(\tau) d \tau \geq 0, \forall s \in R
$$

Obviously, system (2.7) is equivalent to the following system

$$
\left(v_{N t t}-\left(1+k_{0}\right) v_{N x x}-\alpha v_{N x^{4}}-\beta v_{N x^{6}}-\sigma_{1}\left(v_{N x}\right)_{x}, y_{s}\right)=0, s=1,2, \cdots, N .
$$

Multiplying both sides of (2.10) by $2 \dot{\alpha}_{N s}(t)$, summing up for $s=1,2, \cdots, N$, 
and integrating by parts, we obtain

$$
\frac{d}{d t}\left(\left\|v_{N t}\right\|^{2}+\left(1+k_{0}\right)\left\|v_{N x}\right\|^{2}-\alpha\left\|v_{N x x}\right\|^{2}+\beta\left\|v_{N x^{3}}\right\|^{2}+2 \int_{\Omega} \int_{0}^{v_{N x}} \sigma_{1}(s) d s d x\right)=0
$$

When $k_{0}<-1$, by virtue of Lemma 2.1, there is constants $C_{1}>0$ and $C_{2}>0$ such that

$$
\begin{gathered}
\left\|v_{N x}(\cdot, t)\right\|^{2} \leq C_{1}\left\|v_{N}(\cdot, t)\right\|^{2}-\frac{\beta}{4\left(1+k_{0}\right)}\left\|v_{N x^{3}}(\cdot, t)\right\|^{2}, t>0, \\
\left\|v_{N x^{2}}(\cdot, t)\right\|^{2} \leq C_{2}\left\|v_{N}(\cdot, t)\right\|^{2}+\frac{\beta}{4 \alpha}\left\|v_{N x^{3}}(\cdot, t)\right\|^{2}, t>0 .
\end{gathered}
$$

Adding $2\left[1-C_{1}\left(1+k_{0}\right)+C_{2} \alpha\right]\left(v_{N}, v_{N t}\right)$ to the both sides of (2.11), integrating in $[0, t]$, making use of $(2.12),(2.13)$, we get

$$
\begin{aligned}
& \left\|v_{N}(\cdot, t)\right\|^{2}+\left\|v_{N t}(\cdot, t)\right\|^{2}+\frac{\beta}{2}\left\|v_{N x^{3}}(\cdot, t)\right\|^{2}+2 \int_{\Omega} \int_{o}^{v_{N x}} \sigma_{1}(s) d s d x \\
& \leq\left\|v_{0}\right\|^{2}+\left\|v_{1}\right\|^{2}+\beta\left\|v_{0}^{\prime \prime \prime}\right\|^{2}+2 \int_{\Omega} \int_{o}^{v_{0}^{\prime}} \sigma_{1}(s) d s d x \\
& +\left[1-C_{1}\left(1+k_{0}\right)+C_{2} \alpha\right] \int_{0}^{t}\left(\left\|v_{N}(\cdot, \tau)\right\|^{2}+\left\|v_{N t}(\cdot, \tau)\right\|^{2}\right) d \tau, t \in[0, T] .
\end{aligned}
$$

Applying the Gronwall inequality to (2.14), we can obtain (2.9). When $-1 \leq k<0$, adding $2\left(1+C_{2} \alpha\right)\left(v_{N}, v_{N t}\right)$ to the both sides of (2.11), integrating the product over $[0, t]$, making use of (2.13) and the Cauchy inequality and Gronwall inequality, we get (2.9) immediately.

Using (2.9) and the Leray-Schauder fixed point theorem [8], we can prove that the problem (2.7), (2.8) has a solution $\alpha_{N s} \in C^{2}[0, T](s=1,2, \cdots, N)$. Lemma 2.2 is proved.

Lemma 2.3. (Zhou and Fu [9]) Assume that $G(z)$ is a k-times $(k \geq 1)$ continuously differentiable function with respect to variables $z$ and $z \in L^{\infty}\left([0, T] ; H^{k}(\Omega)\right)$. Then

$$
\left\|\frac{\partial^{k}}{\partial x^{k}} G(z)\right\|^{2} \leq C(m, k)\|z(\cdot, t)\|_{H^{k}(\Omega)}^{2},
$$

where $m=\max _{(x, t) \in[0, T] \times \bar{\Omega}}|z(x, t)|, C(m, k)$ is a positive constant depending only on $m$ and $k$.

Lemma 2.4. Assume that the assumption of Lemma 2.2 hold, $\sigma \in C^{k+1}(R)$, $v_{0} \in H^{k+3}(\Omega), v_{1} \in H^{k}(\Omega)$, then there exist the estimates

$$
\begin{gathered}
\left\|v_{N}(\cdot, t)\right\|_{H^{k+3}(\Omega)}^{2}+\left\|v_{N t}(\cdot, t)\right\|_{H^{k}(\Omega)}^{2} \leq C_{2}(T), k \geq 0, \\
\left\|v_{N t t}(\cdot, t)\right\|_{H^{p_{1}}(\Omega)}^{2} \leq C_{3}(T), k=3+p_{1}, p_{1} \geq 0 .
\end{gathered}
$$

Proof: We apply the mathematical induction to prove the estimate $(2.15)$. The estimate (2.9) is the estimate (2.15) when $k=0$. Suppose that when $k=n$, the estimate (2.15) holds. We shall prove that, when $k=n+1$, the estimate $(2.15)$ holds too.

Multiply both sides of (2.7) by $2(-1)^{n+1} \lambda_{s}^{n+1} \alpha_{N s t}(t)$, summing up for 
$s=1,2, \cdots, N$, integrating by parts, we obtain

$$
\begin{aligned}
& \frac{d}{d t}\left(\left\|v_{N x^{n+1} t}(\cdot, t)\right\|^{2}+\left\|v_{N x^{n+2}}(\cdot, t)\right\|^{2}-\alpha\left\|v_{N x^{n+3}}(\cdot, t)\right\|^{2}+\beta\left\|v_{N x^{n+4}}(\cdot, t)\right\|^{2}\right) \\
& =2\left(\sigma\left(v_{N x}\right)_{x^{n+2}}, v_{N x^{n+1} t}\right) .
\end{aligned}
$$

By lemma 2.1, there is a constant $C_{3}>0$, such that

$$
\left\|v_{N x^{n+3}}(\cdot, t)\right\|^{2} \leq C_{3}\left\|v_{N}(\cdot, t)\right\|^{2}+\frac{\beta}{2 \alpha}\left\|v_{N x^{n+4}}(\cdot, t)\right\|^{2}, t>0 .
$$

Adding $2\left(1+C_{3} \alpha\right)\left(v_{N}, v_{N t}\right)$ to the both sides of (2.17), integrating the product over $[0, t]$, Cauchy inequality, Lemma 2.3, (2.9) and (2.18), we have

$$
\begin{aligned}
& \left\|v_{N}(\cdot, t)\right\|^{2}+\left\|v_{N x^{n+1} t}(\cdot, t)\right\|^{2}+\left\|v_{N x^{n+2}}(\cdot, t)\right\|^{2}+\frac{\beta}{2}\left\|v_{N x^{n+4}}(\cdot, t)\right\|^{2} \\
& \leq\left\|v_{0}\right\|^{2}+\left\|v_{1 x^{n+1}}\right\|^{2}+\left\|v_{0 x^{n+2}}\right\|^{2}+\beta\left\|v_{0 x^{n+4}}\right\|^{2}+\int_{0}^{t}\left[C(m, n+2)\left\|v_{N x}(\cdot, \tau)\right\|_{H^{n+2}(\Omega)}^{2}\right. \\
& \left.+\left\|v_{N x^{n+1} \tau}(\cdot, \tau)\right\|^{2}+\left(1+C_{3} \alpha\right)\left(\left\|v_{N}(\cdot, \tau)\right\|^{2}+\left\|v_{N \tau}(\cdot, \tau)\right\|^{2}\right)\right] d \tau,
\end{aligned}
$$

where $m=\max _{(x, t) \in[0, T] \times \bar{\Omega}}\left|v_{N x}(x, t)\right|$. It follows from (2.9), (2.19) and the Gronwall inequality, we get

$$
\left\|v_{N x}^{p_{1} t t}\right\|^{2} \leq\left(\left\|v_{N x^{p_{1}+2}}\right\|+\left\|\sigma\left(v_{N x}\right)_{x^{p_{1}+1}}\right\|+\alpha\left\|v_{N x^{p_{1}}+4}\right\|+\beta\left\|v_{N x^{p_{1}+6}}\right\|\right)\left\|v_{N x^{p_{1} t}}\right\|
$$

Multiply both sides of (2.7) by $(-1)^{p_{1}} \lambda_{s}^{p_{1}} \alpha_{N s t t}(t)$, summing up for $s=1,2, \cdots, N$, integrating by parts, Holder inequality, (2.15), we get

$$
\left\|v_{N x} p_{1 t t}\right\| \leq C_{5}(T), t \in[0, T] .
$$

Theorem 2.1. Under the assumptions of Lemma 2.4, if $k \geq 3$, then the problem (2.1) - (2.3) has a unique generalized global solution $v(x, t)$, which has continuous derivatives $v_{x^{s}}(x, t)(0 \leq s \leq 2)$ and generalized derivatives $v_{x^{s}}(x, t)(0 \leq s \leq 6), v_{x^{s}}(x, t)(0 \leq s \leq 3)$ and $v_{t t}(x, t)$.

Proof: First we give the definition of the generalized solution, which $v(x, t)$ satisfies the identity

$$
\int_{0}^{T} \int_{\Omega}\left[v_{t t}-v_{x x}-\sigma\left(v_{x}\right)_{x}-\alpha v_{x^{4}}-\beta v_{x^{6}}\right] g(x, t) d x d t=0, \forall g(x, t) \in L^{2}\left(Q_{T}\right)
$$

and the periodic boundary value conditions (2.2), (2.3) in the classical sense.

By Lemma 2.4, we have

$$
\begin{gathered}
\left\|v_{N}(\cdot, t)\right\|_{H^{k+3}(\Omega)}^{2}+\left\|v_{N t}(\cdot, t)\right\|_{H^{k}(\Omega)}^{2} \leq C_{2}(T), t \in[0, T], \\
\left\|v_{N t t}(\cdot, t)\right\|_{H^{p_{1}}(\Omega)}^{2} \leq C_{3}(T), t \in[0, T],
\end{gathered}
$$

It follows from Sobolev embedding theorem, when $k=3$, we know

$$
v_{N x^{s}} \in C([0, T] \times \bar{\Omega})(0 \leq s \leq 5), \quad v_{N x^{s} t} \in C([0, T] \times \bar{\Omega})(0 \leq s \leq 2) .
$$

We can select a subsequence of $\left\{v_{N}(x, t)\right\}$ and a function $v(x, t)$ and $N \rightarrow \infty$, the subsequence uniformly converges to the limiting function $v(x, t)$ in $\bar{Q}_{T}$. In fact, $\left\{v_{N}(x, t)\right\}$ is the uniformly bound in $\bar{Q}_{T}$. Meanwhile 


$$
\begin{aligned}
& \left|v_{N}(x+\Delta x, t+\Delta t)-v_{N}(x, t)\right| \\
& \leq\left|v_{N}(x+\Delta x, t+\Delta t)-v_{N}(x, t+\Delta t)\right|+\left|v_{N}(x, t+\Delta t)-v_{N}(x, t)\right| \\
& =\left|v_{N x}\left(x+\theta_{1} \Delta x, t+\Delta t\right)\left\|\Delta x|+| v_{N t}\left(x, t+\theta_{2} \Delta t\right)\right\| \Delta t\right|,
\end{aligned}
$$

where $0<\theta_{1}, \theta_{2}<1, \Delta x$ and $\Delta t$ are the change vectors. Therefore, $\left\{v_{N}(x, t)\right\}$ is equicontinuous in $\bar{Q}_{T}$.

According to Ascoli-Arzela, we can select a subsequence of $\left\{v_{N}(x, t)\right\}$, still denoted by $\left\{v_{N}(x, t)\right\}$, such that there exists a function $v(x, t)$ and $N \rightarrow \infty$, the subsequence $v(x, t)$ uniformly converges to the limiting function $v(x, t)$ in $\bar{Q}_{T}$. The corresponding subsequences $\left\{v_{N x^{s}}(x, t)\right\}(s=1,2)$ also uniformly converges to $v_{x^{s}}(x, t)(s=1,2)$ in $\bar{Q}_{T}$, respectively.

Making use of the weakly compact theorem of the space $L^{2}\left(Q_{T}\right)$, we know that the subsequences $\left\{v_{N x^{s}}(x, t)\right\}(0 \leq s \leq 6),\left\{v_{N x^{s} t}(x, t)\right\}(0 \leq s \leq 3)$ and $\left\{v_{N t t}(x, t)\right\}$ weakly converge to $v_{x^{s}}(x, t)(0 \leq s \leq 6), \quad v_{x^{s} t}(x, t)(0 \leq s \leq 3)$ and $v_{t t}(x, t)$ in $L^{2}\left(Q_{T}\right)$, respectively.

In fact,

$$
\begin{aligned}
& \left|\int_{0}^{T} \int_{\Omega}\left[\sigma\left(v_{N x}\right)_{x}-\sigma\left(v_{x}\right)_{x}\right] g(x, t) d x d t\right| \\
& =\left|\int_{0}^{T} \int_{\Omega}\left[\sigma^{\prime}\left(v_{N x}\right)\left(v_{N x^{2}}-v_{x^{2}}\right)+\sigma^{\prime \prime}\left(v_{x}+\theta_{3}\left(v_{N x}-v_{x}\right)\right) v_{x^{2}}\right] g(x, t) d x d t\right| \\
& \leq \max _{\bar{Q}_{T}}\left|\sigma^{\prime}\left(v_{N x}\right) \| \int_{0}^{T} \int_{\Omega}\left(v_{N x^{2}}-v_{x^{2}}\right) g(x, t) d x d t\right| \\
& \quad+\max _{\bar{Q}_{T}}\left|\sigma^{\prime \prime}\left(v_{x}+\theta_{3}\left(v_{N x}-v_{x}\right)\right) v_{x^{2}} \| \int_{0}^{T} \int_{\Omega}\left(v_{N x}-v_{x}\right) g(x, t) d x d t\right|,
\end{aligned}
$$

where $0<\theta_{3}<1$. So the identity (2.22) holds. Obviously $v(x, t)$ satisfies the periodic boundary value conditions (2.2), (2.3) in the classical sense. Therefore when $k \geq 3$, there exists a generalized global solution $v(x, t)$ of the problem (2.1) - (2.3).

It is easy that we can get the uniqueness of the solution of the periodic boundary value problem (2.1) - (2.3). The Theorem 2.1 is proved.

Theorem 2.2. Under the assumptions of Lemma 2.4, if $k \geq 7$, then the periodic boundary value problem (2.1) - (2.3) has a unique global classical solution $v(x, t)$.

Proof: Differentiating (2.7) with respect to $t$, we have

$$
\left(v_{N t^{3}}-v_{N x x t}-\alpha v_{N x^{4} t}-\beta v_{N x^{6} t}-\sigma\left(v_{N x}\right)_{x t}, y_{s}\right)=0, s=1,2, \cdots, N .
$$

Multiplying both sides of (2.25) by $(-1)^{p_{2}} \lambda_{s}^{p_{2}} \alpha_{N s t^{3}}(t)$, summing up for $s=1,2, \cdots, N$, integrating by parts and using the Holder inequality, combining (2.15), we conclude

$$
\left\|v_{N x} p_{t^{3}}{ }^{3}\right\| \leq C_{7}(T), k=p_{2}+6, p_{2} \geq 0 .
$$

Combining (2.26) and Sobolev embedding theorem, we know that

$$
v_{N x^{s} t^{3}} \in C([0, T] \times \bar{\Omega}), 0 \leq s \leq k-7 .
$$

Using the method of Theorem 2.1, when $k \geq 7$, the periodic boundary value problem (2.1) - (2.3) has a global classical solution $v(x, t)$. It is easy to prove the 
uniqueness of solution for the problem (2.1) - (2.3).

\section{Cauchy Problem (1.6), (1.7)}

Theorem 3.1. Suppose that $\sigma \in C^{k+1}(R), \sigma^{\prime}(s)$ is bounded blow, $v_{0} \in H^{k+3}(R)$, $v_{1} \in H^{k}(R)$. If $k \geq 3\left(k=3+p_{1}, p_{1} \geq 0\right)$, then the Cauchy problem (1.6), (1.7) has a unique global generalized solution $v(x, t)$.

Proof: Let us take a real sequence $\left\{D_{s}\right\}\left(D_{s}>1\right)$ such that $D_{s} \rightarrow \infty$ as $s \rightarrow \infty$. For every $s$, let us construct periodic functions $v_{0 s}(x)$ and $v_{1 s}(x)$ with period $2 D_{s}$ such that

i) $v_{0 s} \in H^{k+3}\left[-D_{s}, D_{s}\right], v_{1 s} \in H^{k}\left[-D_{s}, D_{s}\right]$;

ii) $v_{0 s}(x)=v_{0}(x), v_{1 s}(x)=v_{1}(x)$ for $x \in\left[-\left(D_{s}-1\right), D_{s}-1\right]$ and

$$
\begin{aligned}
&\left\|v_{0 s x^{i}}\right\|_{L^{2}\left[-D_{s}, D_{s}\right]} \leq\left\|v_{0 x^{i}}\right\|_{L^{2}(R)}, i=0,1, \cdots, k+3, \\
&\left\|v_{1 s x^{i}}\right\|_{L^{2}\left[-D_{s}, D_{S}\right]} \leq\left\|v_{1 x^{i}}\right\|_{L^{2}(R)}, i=0,1, \cdots, k+1 .
\end{aligned}
$$

we consider the following periodic boundary value problem

$$
\begin{gathered}
v_{t t}=v_{x x}+\sigma\left(v_{x}\right)_{x}+\alpha v_{x^{4}}+\beta v_{x^{6}}, \\
v_{x}(x, t)=v_{x}\left(x+2 D_{s}, t\right), \\
v(x, 0)=v_{0 s}(x), v_{t}(x, 0)=v_{1 s}(x) .
\end{gathered}
$$

Let $\left\{y_{n}(x)\right\}=\left\{\frac{1}{\sqrt{2 D}}, \frac{1}{\sqrt{D}} \cos \delta_{n} x, \frac{1}{\sqrt{D}} \sin \delta_{n} x, n=1,2, \cdots\right\}$ be the orthogonal base in $L^{2}\left(\Omega_{s}\right)$ composed of eigenfunctions of eigenvalue problem

$$
\begin{aligned}
& y^{\prime \prime}+\lambda y=0, x \in \Omega_{s}, \\
& y_{x}(x)=y_{x}\left(x+2 D_{s}\right)
\end{aligned}
$$

corresponding to eigenvalue $\lambda_{i}=\delta_{i}^{2}=\left(\frac{i \pi}{D_{s}}\right)^{2}(i=1,2, \cdots)$, where $\Omega_{s}=\left(-D_{s}, D_{s}\right)$.

Suppose that the Galerkin approximate solution of (3.1)-(3.3) is

$$
v_{N_{s}}(x, t)=\sum_{i=1}^{N_{s}} \alpha_{N_{s} i}(t) y_{i}(x)
$$

where $\alpha_{N_{s} i}(t)$ are the undermined functions.

Let $v_{N_{s}}(x, t)$ satisfy the following equation and conditions

$$
\begin{gathered}
v_{N_{s} t t}=v_{N_{s} x x}+\sigma\left(v_{N_{s} x}\right)_{x}+\alpha v_{N_{s} x^{4}}+\beta v_{N_{s} x^{6}}, \\
v_{N_{s} x}(x, t)=v_{N_{s} x}\left(x+2 D_{s}, t\right), \\
v_{N_{s}}(x, 0)=v_{0 s}(x), v_{N_{s} t}(x, 0)=v_{1 s}(x) .
\end{gathered}
$$

By the same method as in the estimates (2.15), (2.16), we have

$$
\left\|v_{N_{s}}\right\|_{H^{k+3}\left(\Omega_{s}\right)}+\left\|v_{N_{s} t}\right\|_{H^{k}\left(\Omega_{s}\right)}+\left\|v_{N_{s} t^{2}}\right\|_{H^{p_{1}}\left(\Omega_{s}\right)} \leq C_{8}(T), t \in[0, T]
$$

where and in the sequel $C_{i}(T)(i=8,9, \cdots)$ are constants independent of $N_{s}$ and $D_{s}$. By the Sobolev imbedding theorem when $k=4$, we get 


$$
\begin{gathered}
\left\|v_{N_{s}}\right\|_{H^{6}\left(\Omega_{s}\right)}+\left\|v_{N_{s} t}\right\|_{H^{3}\left(\Omega_{s}\right)}+\left\|v_{N_{s} t^{2}}\right\|_{L^{2}\left(\Omega_{s}\right)} \leq C_{9}(T), t \in[0, T], \\
\left\|v_{N_{s}}\right\|_{C^{5}\left(\bar{\Omega}_{s}\right)}+\left\|v_{N_{s}} t\right\|_{C^{2}\left(\bar{\Omega}_{s}\right)} \leq C_{10}(T), t \in[0, T] .
\end{gathered}
$$

By virtue of (3.9) and Ascoli-Arzela theorem, we can select from $\left\{v_{N_{s}}(x, t)\right\}$ a subsequence, still denoted by $\left\{v_{N_{s}}(x, t)\right\}$, such that when $N_{s} \rightarrow \infty$, $\left\{v_{N_{s} x^{k}}(x, t)\right\}(0 \leq k \leq 2)$ uniformly converge to limiting functions $v_{s x^{k}}(x, t)(0 \leq k \leq 2)$ in $\bar{\Omega}_{s} \times[0, T]$, respectively.

The estimates (3.8) still holds for the above subsequence $\left\{v_{N_{s}}(x, t)\right\}$. Hence, we can select from $\left\{v_{N_{s}}(x, t)\right\}$ a subsequence, still denoted by $\left\{v_{N_{s}}(x, t)\right\}$, such that when $N_{s} \rightarrow \infty$, the subsequences $\left\{v_{N_{s} k^{k}}(x, t)\right\}(0 \leq k \leq 6)$,

$\left\{v_{N_{s} k_{t} t^{k}}(x, t)\right\}(0 \leq k \leq 3)$ and $\left\{v_{N_{s} t t}(x, t)\right\}$ weakly converge to limiting functions $v_{s x^{k}}(x, t)(0 \leq k \leq 6), v_{s x^{k} t}(x, t)(0 \leq k \leq 3)$ and $v_{s t t}(x, t)$ in $L^{2}\left((0, T) ; L^{2}\left(\Omega_{s}\right)\right)$, respectively.

From the corollary of the resonance theorem [10], it follows that the estimates (3.8), (3.9) still hold for $v_{s}(x, t)$, which is the generalized solution of the problem (3.1)-(3.3). Using Ascoli-Arzela theorem, we can select from $\left\{v_{s}(x, t)\right\}$ a subsequence still denoted by $\left\{v_{s}(x, t)\right\}$, such that when $s \rightarrow \infty$, the subsequences $\left\{v_{s x^{k}}(x, t)\right\}(0 \leq k \leq 2)$ uniformly converge to limiting functions $v_{x^{k}}(x, t)(0 \leq k \leq 2)$ in any domain $\{-L \leq x \leq L, 0 \leq t \leq T\}$, respectively.

It follows from (3.8) that we can select from $v_{s}(x, t)$ a subsequence, still denoted by $v_{s}(x, t)$, such that when $s \rightarrow \infty$, in $L^{2}\left((0, T) ; L^{2}(-L, L)\right)$, the subsequences $\left\{v_{s x^{k}}(x, t)\right\}(0 \leq k \leq 6), \quad\left\{v_{s x^{k}}(x, t)\right\}(0 \leq k \leq 6) \quad$ and $\quad\left\{v_{s t t}(x, t) \quad\right.$ weakly converge to limiting functions $\left\{v_{x^{k}}(x, t)\right\}(0 \leq k \leq 6), \quad\left\{v_{x^{k}}(x, t)\right\}(0 \leq k \leq 3)$ and $\left\{v_{t t}(x, t)\right\}$, respectively. The obtained limiting function $v(x, t)$ is just the global generalized solution of the auxiliary problem (1.6), (1.7).

Clearly, the generalized solution of the auxiliary problem (1.6), (1.7) is also unique. Therefore when $k \geq 3$, the Cauchy problem (1.6), (1.7) has a unique global generalized solution.

Theorem 3.2. Assume that the assumptions of Theorem 3.1 hold, If $k \geq 7\left(k=6+p_{2}, p_{2} \geq 0\right)$, then the Cauchy problem (1.6), (1.7) has a unique global classical solution $v(x, t)$.

Proof: Combining the estimates (2.15), (2.16) with (2.35), we obtain

$$
\left\|v_{N_{s}}\right\|_{H^{k+3}\left(\Omega_{s}\right)}+\left\|v_{N_{s} t}\right\|_{H^{k}\left(\Omega_{s}\right)}+\left\|v_{N_{s} t^{2}}\right\|_{H^{p_{1}}\left(\Omega_{s}\right)}+\left\|v_{N_{s} t^{3}}\right\|_{H^{p_{2}\left(\Omega_{s}\right)}} \leq C_{11}(T), t \in[0, T]
$$

By the Sobolev imbedding theorem when $k=7$, we get

$$
\begin{gathered}
\left\|v_{N_{s}}\right\|_{H^{10}\left(\Omega_{s}\right)}+\left\|v_{N_{s} t}\right\|_{H^{7}\left(\Omega_{s}\right)}+\left\|v_{N_{s} t^{2}}\right\|_{H^{4}\left(\Omega_{s}\right)}+\left\|v_{N_{s} t^{3}}\right\|_{H^{1}\left(\Omega_{s}\right)} \leq C_{12}(T), \\
\left\|v_{N_{s}}\right\|_{C^{9}\left(\bar{\Omega}_{s}\right)}+\left\|v_{N_{s} t}\right\|_{C^{6}\left(\bar{\Omega}_{s}\right)}+\left\|v_{N_{s} t}\right\|_{C^{3}\left(\bar{\Omega}_{s}\right)}+\left\|v_{N_{s} t^{3}}\right\|_{C\left(\bar{\Omega}_{s}\right)} \leq C_{13}(T) .
\end{gathered}
$$

Using the method of Theorem 3.1, when $k \geq 7$, the Cauchy problem (1.6), (1.7) has a global classical solution. It is easy to prove the uniqueness of solution 
for the problem (1.6), (1.7).

\section{Cauchy Problem (1.1), (1.2)}

Lemma 4.1. [11] Suppose that $s=m+\frac{1}{2}+\lambda, \lambda \in(0,1), m \in Z_{+}$, then $H^{s}(R)$ may be embedded into $C^{m+\lambda}(R)$, and for any $u \in H^{s}(R)$, we have

$$
\left|\frac{d^{k}}{d x^{k}} u(x)\right| \rightarrow 0(|x| \rightarrow \infty), \forall k \in Z_{+}, 0 \leq k \leq m,
$$

where $Z_{+}$is a set of nonnegative integers.

Theorem 4.1. Suppose that $\sigma \in C^{k+2}(R), \sigma^{\prime}(s)$ is bounded blow, $u_{0} \in H^{k+2}(R), u_{1} \in H^{k-1}(R)$. If $k-1 \geq 3\left(k=3+p_{1}, p_{1} \geq 0\right)$, then the Cauchy problem (1.1), (1.2) has a unique global generalized solution $u(x, t)$.

Proof: Differentiating (3.4) and (3.6) with respect to $x$, we have

$$
\begin{gathered}
v_{N_{s} x t t}=v_{N_{s} x^{3}}+\sigma\left(v_{N_{s} x}\right)_{x x}+\alpha v_{N_{s} x^{5}}+\beta v_{N_{s} x^{7}}, \\
v_{N_{s} x}(x, 0)=v_{0 s x}(x), v_{N_{s} x t}(x, 0)=v_{1 s x}(x) .
\end{gathered}
$$

Let

$$
v_{N_{s} x}(x, t)=u_{N_{s}}(x, t) .
$$

Substituting (4.3) into (4.1), (3.5) and (4.2), we obtain

$$
\begin{gathered}
u_{N_{s} t t}=u_{N_{s} x x}+\sigma\left(u_{N_{s}}\right)_{x x}+\alpha u_{N_{s} x^{4}}+\beta u_{N_{s} x^{6}}, \\
u_{N_{s}}(x, t)=u_{N_{s}}\left(x+2 D_{s}, t\right), \\
u_{N_{s}}(x, 0)=u_{0 N_{s}}(x), u_{N_{s} t}(x, 0)=u_{1 N_{s}}(x) .
\end{gathered}
$$

By using the change (4.3), it follows from (3.7) that

$$
\left\|u_{N_{s}}\right\|_{H^{k+2}\left(\Omega_{s}\right)}+\left\|u_{N_{s} t}\right\|_{H^{k-1}\left(\Omega_{s}\right)}+\left\|u_{N_{s} t^{2}}\right\|_{H^{p^{1}-1}\left(\Omega_{s}\right)} \leq C_{14}(T), t \in[0, T] .
$$

From (4.7) and the Sobolev imbedding theorem, we know that

$$
\left\|u_{N_{s}}\right\|_{C^{k+1}\left(\bar{\Omega}_{s}\right)}+\left\|u_{N_{s}}\right\|_{C^{k-2}\left(\bar{\Omega}_{s}\right)}+\left\|u_{N_{s} t t}\right\|_{C^{p^{1}-2}\left(\bar{\Omega}_{s}\right)} \leq C_{15}(T), t \in[0, T] .
$$

By using the same method as in Section 3, it follows from (4.7) and (4.8) that, when $k \geq 4$, the Cauchy problem (1.1), (1.2) has a generalized global solution $u(x, t)$.

It is easy that, we prove the uniqueness of solution for the problem (1.1), (1.2). Hence, Theorem 4.1 is proved.

Theorem 4.2. Assume that the assumptions of Theorem 4.1 hold, If $k-1 \geq 7$ $\left(k=6+p_{2}, p_{2} \geq 0\right)$, then the Cauchy problem (1.1), (1.2) has a unique global classical solution $u(x, t)$.

Proof: By virtue of Theorem 3.2, when $k \geq 8$, the problem (1.6), (1.7) has a unique global classical solution $v(x, t)$. Differentiating Equation (1.6), (1.7) with $x$ and substituting $v_{x}(x, t)=u(x, t)$ into this equation and

$v_{0 x}(x)=u_{0}(x), v_{1 x}(x)=u_{1}(x)$ into the obtained initial value condition, we get $u(x, t)$ is the classical global solution of (1.1), (1.2). The proof is completed. 


\section{Acknowledgements}

This work is supported by the National Natural Science Foundation of China (NSFC) under Grant No. 11671367 and Natural Science Foundation of Henan Province, China under Grant No. 152300410227.

\section{References}

[1] Darapi, P. and Hua, W. (1999) A Numerical Method for Solving an Ill-Posed Boussinesq Equation Arising in Water Waves and Nonlinear Lattices. Applied Mathematics and Computation, 101, 159-207. https://doi.org/10.1016/S0096-3003(98)10070-X

[2] Dash, R.K. and Daripa, P. (2002) Analytical and Numerical Studies of a Singularly Perturbed Boussinesq Equation. Applied Mathematics and Computation, 126, 1-30. https://doi.org/10.1016/S0096-3003(01)00166-7

[3] Darapi, P. and Hua, W. (2001) Weakly Non-Local Solitary Wave Solutions of a Singularly Perturbed Boussinesq Equation. Mathematics and Computers in Simulation, 55, 393-405. https://doi.org/10.1016/S0378-4754(00)00288-3

[4] Feng, Z.S. (2003) Traveling Solitary Wave Solutions to the Generalized Boussinesq Equation. Wave Motion, 37, 17-23. https://doi.org/10.1016/S0165-2125(02)00019-7

[5] Song, C., Li, H. and Li, J. (2013) Initial Boundary Value Problem for the Singularly Perturbed Boussineaq-Type Equation. Discrete and Continuous Dynamical Systems, 709-717.

[6] Song, C., Li, J. and Gao, R. (2014) Nonexistence of Global Solutions to the Initial Boundary Value Problem for the Singularly Perturbed Sixth Order Boussinesq Equation. Hindawi Publishing Corporation, Journal of Applied Mathematics.

[7] Admas, R.A. (1975) Sobolev Space. Academic Press, New York.

[8] Friedman, A. (1964) Partial Differential Equation of Parabolic Type. Prentice Hall, Eagliweed Cliffs.

[9] Zhou, Y.L. and Fu, H.Y. (1983) Nonlinear Hyperbolic Systems of Higher Order Generalized Sine-Gordon Type. Acta Mathematica Sinica, 26, 234-249.

[10] Yosida, K. (1980) Functional Analysis. 6th edition, Springer Verlag, Berlin.

[11] Wang, Y.D. (1989) L2 Theory of Partial Differential Equations. Peking University Press, Beijing. 
Submit or recommend next manuscript to SCIRP and we will provide best service for you:

Accepting pre-submission inquiries through Email, Facebook, LinkedIn, Twitter, etc. A wide selection of journals (inclusive of 9 subjects, more than 200 journals)

Providing 24-hour high-quality service

User-friendly online submission system

Fair and swift peer-review system

Efficient typesetting and proofreading procedure

Display of the result of downloads and visits, as well as the number of cited articles Maximum dissemination of your research work

Submit your manuscript at: http://papersubmission.scirp.org/

Or contact jamp@scirp.org 